\title{
L'évolution de la rage au Canada
}

\author{
Filejski $\mathrm{C}^{1 *}$
}

\section{Résumé}

Les programmes de prévention et de contrôle de la rage au Canada se sont révélés très efficaces au cours des dernières décennies et ont fortement réduit les cas de rage chez les animaux terrestres et les humains. À ce jour, la réussite de la gestion et de la prévention de la rage n'a toutefois pas pu éliminer le besoin de nous soumettre à des programmes continus de prévention et de contrôle de la rage.

Ce numéro du Relevé des maladies transmissibles au Canada (RMTC) propose un aperçu des tendances et défis récents et émergents relativement à la rage au Canada et étudie la justification visant à maintenir nos programmes liés à la rage et à les compléter par de nouvelles approches novatrices. Les articles de ce numéro couvrent de nombreux sujets, notamment la préparation et l'intervention en cas d'incursions renouvelées d'une variante du virus de la rage du raton laveur, comment régler le problème que pose le déplacement des chiens du Nord au Sud du Canada et la manière dont le Plan de gestion de la rage au Canada est révisé et mis à jour pour répondre à ces problèmes.

La rage au Canada évolue, mais elle ne disparaît pas. Il en va de même pour nos politiques et programmes de prévention et de contrôle de la rage.

\begin{abstract}
Affiliation
${ }^{1}$ Vétérinaire de santé publique, ministère de la Santé et des Soins de longue durée de l'Ontario, Toronto (Ontario)
\end{abstract}

${ }^{\star}$ Correspondance : catherine. filejski@ontario.ca

Citation proposée : Filejski C. L'évolution de la rage au Canada. Relevé des maladies transmissibles au Canada 2016;42:132-4. https://doi.org/10.14745/ccdr.v42i06a01f

\section{Introduction}

Après avoir réussi, pendant des décennies, à prévenir les cas de rage chez les humains et à fortement réduire la rage chez les populations de mammifères terrestres au Canada, des questions ont commencé à être soulevées concernant la valeur et l'utilité réelles découlant de la continuité des programmes de prévention et de contrôle de la rage. À une époque de restrictions budgétaires et de priorités concurrentes en matière de maladies infectieuses, avons-nous réellement besoin de continuer d'enquêter sur toutes les morsures et les expositions pouvant entraîner la transmission de la rage aux humains? Après tout, les cas de rage chez les humains restent exceptionnellement rares au Canada. Au cours de la première décennie du XXIe siècle, seuls trois cas contractés sur le territoire ont été observés et tous étaient dus à des souches de rage chez la chauve-souris (1). Dans le cas de la faune, l'Ontario (la province connue autrefois pour être la « capitale nord-américaine de la rage ") a réussi à éradiquer la souche de rage du raton laveur de ses régions de l'Est en 2005. En 2008, il a été déclaré que la rage du raton laveur avait disparu de la province (2) et la province n'a déclaré que deux cas de rage terrestre due à une souche de rage du renard en 2011 (3). Dans de nombreux cas, une certaine complaisance concernant la prévention et le contrôle de la rage a commencé à s'installer.

\section{Nouvelles tendances et nouveaux défis}

Malheureusement, dès 2012, de nouvelles tendances et de nouveaux défis concernant la prévention et la maîtrise de la rage au Canada ont commencé à apparaître et aujourd'hui, la situation de la rage au Canada est bien moins encourageante que ce qu'elle semblait être il y a quatre ans. Ce numéro spécial du Relevé des maladies transmissibles au Canada ayant pour thème la rage explore les changements récents et les nouvelles tendances en matière de risque découlant de la rage dans un certain nombre de provinces.

\section{Retour des souches de rage du raton laveur}

La rage continue de poser un risque important pour la santé du public et des animaux au Canada pour un certain nombre de raisons. Tout d'abord, bien que les mesures de prévention et de maîtrise chez la faune aient fortement réduit le nombre de cas chez les animaux dans le Sud du Canada, la rage reste un problème important aux États-Unis et la menace que représentent les incursions du virus de la rage au-delà de la frontière dans les provinces canadiennes reste réelle et constante. Dans ce numéro, Stevenson et al. présentent un rapport sur trois incursions renouvelées de souche de rage du raton laveur qui s'est de nouveau retrouvée au Nouveau-Brunswick, au Québec et en Ontario au cours des deux dernières années (4). 


\section{Persistance des souches de rage du renard arctique}

Malgré le peu de cas de rage terrestre dans le Sud du Canada, la variante de la souche de rage du renard arctique reste endémique dans le Nord du Canada et son élimination semble peu probable. Dans le Nord, les populations de renards arctiques ont tendance à passer par des cycles d'éclosions de rage qui peuvent devenir suffisamment importantes pour se propager et toucher les populations de chiens dans les collectivités du Nord et les populations de renards roux dans le Sud. La migration de la souche du virus de la rage du renard entre diverses populations d'animaux a eu, en fin de compte, des conséquences importantes par le passé. Dans le milieu des années 1950, la propagation des éclosions de rage du renard arctique dans le Nord dans des populations de renards roux plus au Sud a conduit à la migration de la rage du renard dans le Sud de l'Ontario et du Québec (5).

\section{Problèmes découlant des translocations}

Plus récemment, au cours de l'hiver 2011-2012, des événements liés à une éclosion importante de rage du renard arctique dans le Nord du Québec (Nunavik) et dans l'Ouest du Labrador, ont conduit à l'identification d'une nouvelle préoccupation croissante concernant la rage au Canada : l'introduction de la rage dans des zones urbaines fortement peuplées en raison de la translocation (mouvement rapide sur de grandes distances) des chiens venant du Nord. Au cœur de l'éclosion de rage de 2011-2012, une organisation de protection des animaux a sauvé des chiens errants du Nord du Québec et leur a trouvé de nouveaux foyers dans le Sud. Ces activités ont entraîné la translocation de chiots porteurs de la rage du Nunavik vers Montréal en janvier 2012 (6). Au lieu d'être un incident isolé, ce premier cas était la première indication de problèmes se profilant à l'horizon, car un flot grandissant de chiens sans propriétaires ont été déplacés des collectivités éloignées du Nord dans de nouveaux foyers d'adoption dans le Sud. Dans le deuxième article de ce numéro, Curry et al. rendent compte de deux cas subséquents concernant la translocation de chiens porteurs de la rage dans deux provinces différentes (7).

\section{Problème mondial}

L'importation ou la translocation de chiens porteurs de la rage se sont également produites aux États-Unis et en Europe et ont entraîné des préoccupations accrues de la part des autorités de santé publique. Les exigences relatives aux certificats de vaccination contre la rage pour les chiens traversant les frontières internationales (à titre de prévention de la propagation de la rage) sont sabotées. En janvier 2014, la National Association of State Public Health Veterinarians a fortement encouragé les Centers for Disease Control and Prevention (CDC) des États-Unis à réviser et à élaborer davantage les règlements existants sur l'importation concernant les chiens, qui remontaient aux années 1950, car ils n'ont pas réussi à « réduire adéquatement le risque qu'un animal porteur de la rage entre sur le territoire américain » (correspondance personnelle du président de la National Association of State Public Health Veterinarians, Inc. [NASPHV] et le président du Rabies Compendium Committee de la NASPHV au directeur des CDC des États-Unis, 14 janvier 2014. http://tinyurl.com/lpmou79). En mai 2014, les CDC des États-Unis ont publié le document Health
Alert on Imported Dogs with Questionable Documentation relevant des renseignements sur les certificats de vaccination douteux d'un nombre croissant de chiens qui ont été importés aux États-Unis depuis des pays où la rage est endémique (8). Leur préoccupation était justifiée. En mai 2015, un chien importé aux États-Unis en provenance d'Égypte a reçu un diagnostic de rage et il s'est avéré qu'il était entré aux États-Unis avec un certificat de vaccination contre la rage falsifié (9). Les préoccupations croissantes relatives aux risques d'importation (ou de translocation) d'animaux domestiques qui n'ont pas été adéquatement vaccinés contre la rage sont désormais de plus en plus exprimées, non seulement au Canada et aux États-Unis, mais aussi en Europe et au Royaume-Uni (10).

\section{Approches novatrices}

Le Canada est confronté à des problèmes semblables concernant l'importation de chien au pays. En outre, d'après Curry et al., la translocation pose un problème supplémentaire, car le transport de l'animal au sein des frontières du pays ne requiert pas la présentation de certificats de vaccination contre la rage. Réduire le risque de rage dû à la translocation de chiens du Nord au Sud au Canada nécessitera l'établissement de solutions novatrices pour limiter le nombre de populations de chiens ( $p$. ex. réduire le flot de chiens ou l'arrêter complètement) et augmenter le taux de vaccination contre la rage chez le chien dans les collectivités éloignées du Nord. Lidstone-Jones et Gagnon font un rapport sur une approche novatrice liée à la gestion des populations de chiens, qui est mise à l'essai le long des littoraux de la baie James et de la baie d'Hudson en Ontario, à l'aide d'un contraceptif injecté chez les chiennes (11). Bien que cette méthode ne soit traditionnellement pas considérée comme une mesure de prévention et de contrôle de la rage, il devient de plus en plus évident que les stratégies efficaces et durables de gestion des populations de chiens dans le Nord constituent un volet important de la prévention de la rage, aussi bien pour les collectivités canadiennes du Nord que du Sud.

\section{Coordination intergouvernementale}

Il est probable que la rage de la chauve-souris restera endémique au Canada dans un avenir prévisible. Ce fait, ainsi que la réapparition de la rage du raton laveur au Nouveau-Brunswick, au Québec et en Ontario, le nombre croissant de chiens porteurs de la rage ayant fait l'objet d'une translocation et les défis liés à la surpopulation de chiens auxquels sont confrontées nos collectivités éloignées du Nord au Canada, permettent tous d'insister sur l'importance de rester vigilants quant à la prévention et au contrôle de la rage.

La complexité d'une gestion réussie de la rage à l'échelle nationale se reflétait dans l'élaboration du Plan canadien de gestion de la rage. À l'origine, le Plan a été signé par l'Agence canadienne d'inspection des aliments et l'Agence de la santé publique du Canada en 2009 et propose un aperçu des rôles, des responsabilités et des activités en lien avec la rage à l'échelle du pays. Toutefois, les changements dans le programme fédéral contre la rage qui ont été mis en œuvre en 2014 ont fortement modifié ces rôles, responsabilités et activités au Canada. Dans le dernier article de ce numéro, Tataryn et Buck fournissent un 
aperçu de la manière dont le Plan canadien de gestion de la rage est révisé et mis à jour pour refléter ces changements ainsi que les nouveaux défis auxquels est confronté le Canada en matière de prévention et de contrôle de la rage (12).

\section{Conclusion}

La nouvelle réalité est que la translocation d'animaux, qu'ils soient sauvages ou domestiqués, peut changer radicalement la situation de risque local de la rage dans une région d'un jour à l'autre. Cela signifie que les méthodes traditionnelles permettant de mesurer le risque de rage, fondées exclusivement sur le nombre de cas de rage à l'échelle locale, doivent être équilibrées et complétées par d'autres facteurs, notamment les déplacements des animaux dans une région et en dehors de celle-ci. De nouvelles approches sont nécessaires pour aborder la nouvelle réalité de la rage au Canada, comme la mise en place de nouveaux vaccins oraux antirabiques pour la faune, une collaboration intergouvernementale plus efficace et des stratégies novatrices de gestion des populations de chiens dans le Nord. La rage au Canada évolue, mais elle ne disparaît pas. II en va de même pour nos politiques et programmes de prévention et de contrôle de la rage.

\section{Remerciements}

La $D^{\text {re }}$ Catherine Filejski était une rédactrice invitée pour ce numéro et se récuse des décisions de rédaction relatives au présent article. Les décisions de rédaction ont été prises par la

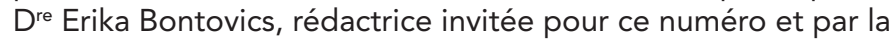
$\mathrm{D}^{\mathrm{re}}$ Patricia Huston, rédactrice en chef du RMTC.

\section{Conflit d'intérêts}

Aucun.

\section{Références}

1. National Advisory Committee on Immunization (NACl). Rabies vaccine. In: Canadian immunization guide. Ottawa: Public Health Agency of Canada; 2014. (Disponible en français : www.phac-aspc. gc.ca/publicat/cig-gci/index-fra.php).

2. Ontario Ministry of Natural Resources. Rabies reporter, Summer 2008. MNR Publication 51709, Vol 19(2), 2008.

3. Ontario Ministry of Natural Resources. Rabies reporter, Oct-Dec 2011. MNR Publication 51709, Vol 22(4), 2011.

4. Stevenson B, Goltz J, Massé A. Préparation et intervention suite aux cas récents de rage du raton laveur au Canada. Relevé des maladies transmissibles au Canada 2016;42:140-4.

5. Lagacé F. Historique de la rage au Québec de 1958 à 1997. Le Médecin Vétérinaire du Québec 2008;28(3).

6. Ministère de la Santé et des Services Sociaux du Québec. Vigie-interventions : Rage chez des chiots. Flash Vigie Feb 2012;7(2).

7. Curry P, Kostiuk D, Werker DH, Baikie M, Ntiamoah W, Atherton F. Propagation de la rage par des chiens en provenance du Nunavut. Relevé des maladies transmissibles au Canada 2016;42:135-9.

8. CDC warns of flawed rabies certificates. JAVMA Jul 2014;245(1). https://www.avma.org/News/JAVMANews/Pages/140701k.aspx.

9. Sinclair $D$, et al. Rabies in a dog imported from Egypt with a falsified rabies vaccination certificate: Virginia, 2015. MMWR Dec 2015;64(49).

10. Ford E. Government urged to review rabies risk. BBC News. 2013 Oct 20. http://www.bbc.com/news/uk-24569593.

11. Lidstone-Jones C, Gagnon R. Innovation dans la prévention et la maîtrise de la rage dans le Nord : le project pilote de gestion de la population de chiens dans la zone relevant de l'autorité sanitaire de la région de Weeneebayko. Relevé des maladies transmissibles au Canada 2016;42:145-50.

12. Tataryn J, Buck PA. Le plan canadien de gestion de la rage : une approche intégrée de coordination des activités de lutte contre la rage au Canada. Relevé des maladies transmissibles au Canada 2016;42:151-2. 